\title{
Investigation of Closing Surge in Shinkansen Power System and Proposal of a Novel Power Electronics Application for Changeover Section
}

\author{
Hitoshi Hayashiya Member (JR East, h-hayashiya@jreast.co.jp) \\ Yoshinobu Ueda Member (Meidensha Co.) \\ Koji Ajiki Member (RTRI) \\ Hideo Watanabe Member (Meidensha Co.) \\ Masato Ando Member (JR East) \\ Makoto Nakajima Non-member (JR East)
}

Keywords: changeover section, closing surge, Shinkansen, AT feeding system

In this paper, the closing surge phenomena of the Shinkansen changeover section is described based on the detail measurements on the operational substations. In Shin-Tabata substation, the frequent work of the lightning arrester for changeover section is observed and it becomes the slight anxiety for daily maintenance. Fig. 1 shows the measured discharge current of the lightning arrester. The example of the voltage waveform which causes the arrester work is also shown in Fig. 2. It is clarified that the closing surge up to $100 \mathrm{kV}$ leads to the $100 \mathrm{~A}$ discharge current.

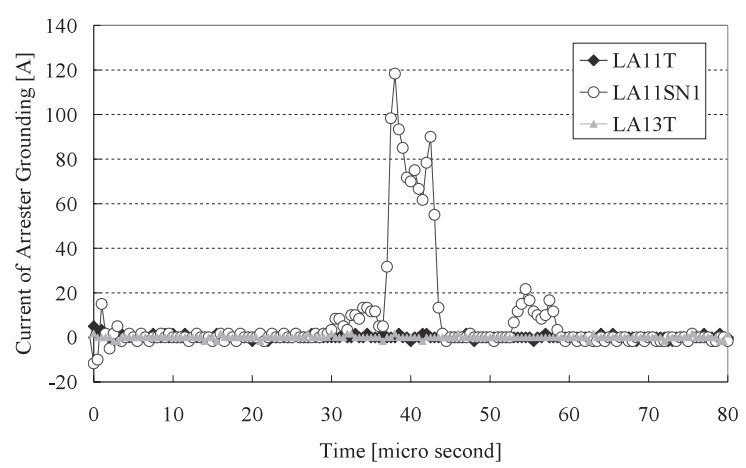

Fig. 1. Waveform of the discharge current of LA

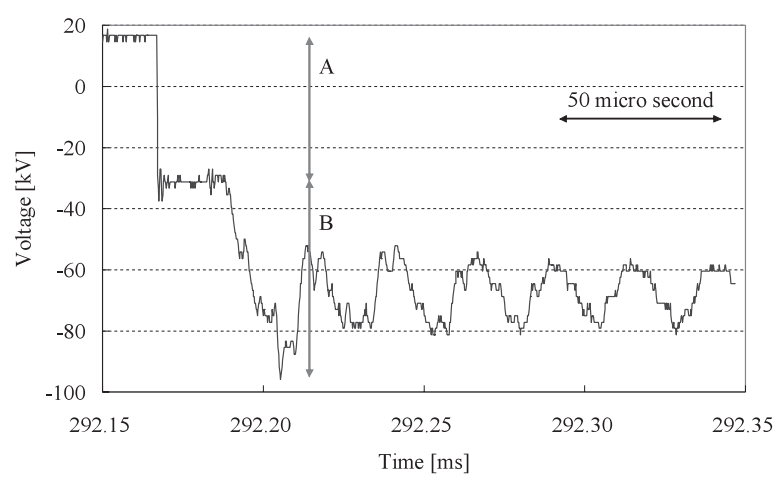

Fig. 2. Waveform of the voltage at changeover section
Judging from the waveform shown in Fig. 2 and the other measured data, the over-voltage of the changeover section is caused by the traveling of the closing surge which is reflected and doubled at the open terminal of the changeover section conductor. The reflected voltage comes back to the switchgear 38 micro second after the first electrical contact of the switchgear.

Following the description of the surge phenomena, a novel power supply system for Shinkansen changeover section (CSPS: changeover section power system) is proposed as one of the solutions to avoid such a over-voltage. By applying this system, the Shinkansen train is able to pass the changeover section without electrically transient phenomena including the closing surge and instantaneous power failure. Fig. 3 shows the configuration of the CSPS.

Three special control methods, those are voltage and phase shift control, load transfer control and output restriction control, are proposed to make the inverter output stable. Its efficiency is conformed by both the computer simulation and the mini-model experiments. Such a power electronics application will be one of the idealistic devices for the advanced power system of the high speed railway transportation in the future.

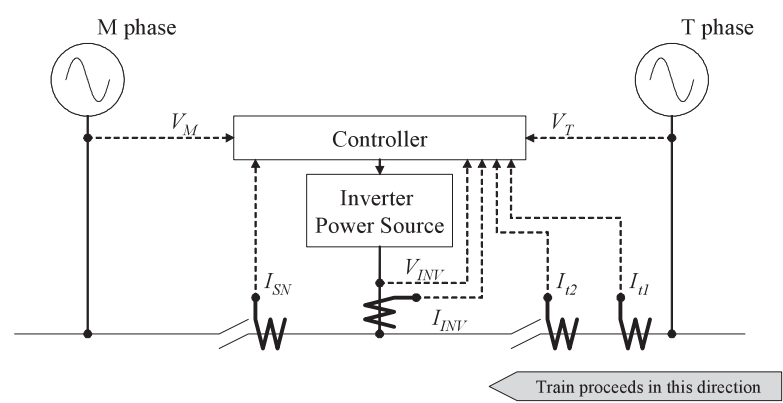

Fig. 3. Configuration of the proposed CSPS 


\title{
Investigation of Closing Surge in Shinkansen Power System and Proposal of a Novel Power Electronics Application for Changeover Section
}

\author{
Hitoshi Hayashiya* Member \\ Yoshinobu Ueda** Member \\ Koji Ajiki*** Member \\ Hideo Watanabe ${ }^{* *}$ Member \\ Masato Ando* Member \\ Makoto Nakajima* Non-member
}

\begin{abstract}
The transient surge measurements at the changeover sections of Shinkansen power feeding system were carried out in some substations and sectioning posts. The detail of the surge voltage phenomena was investigated and the reason of the frequent discharge of the lightning arrester was clarified. As one of the solutions to avoid such a severe transient phenomena, a novel power supply system for a changeover section substitution for the conventional system with a pair of the vacuum switchgear was proposed. To realize a stable running through the changeover section where two power sources with phase difference are faced, some special control methods were introduced and their validities are confirmed by not only the computer simulations but also the mini-model experiments.
\end{abstract}

Keywords: changeover section, closing surge, vacuum switchgear, Shinkansen, AT feeding system, changeover section power system (CSPS), inverter

\section{Introduction}

In some substations and sectioning posts of Shinkansen power system in JR East area, the electrical transient phenomena when the Shinkansen train passes through the changeover section are reported during the last a few years. At Shin-Tabata substation on Tohoku Shinkansen line, for example, the discharge counter of the lightning arrester for changeover section acts frequently when the changeover section works. The average action frequency is about 5 times per a day and it becomes something anxiety for daily maintenance.

In this paper, the closing surge phenomena of the Shinkansen changeover section is described based on the detail measurements on the practical substations and sectioning posts. Following the description of the phenomena, a novel power supply system for Shinkansen changeover section (CSPS: Changeover Section Power System) is proposed. By applying this system to changeover section, the train is able to pass the changeover section without electrically transient phenomena including the closing surge, opening surge and instantaneous power failure. The control characteristics of CSPS are confirmed by not only power electronics circuit

\footnotetext{
* East Japan Railway Company, h-hayashiya@jreast.co.jp Technical Center, R\&D Center of JR East Group 2-0, Nisshin-cho, Kita-ku Saitama-shi, Saitama 331-8513, JAPAN

** Meidensha Corporation, ueda-yo@mb.meidensha.co.jp Riverside Building

36-2, Nihonbashi Hakozakicho, Chuo-ku, Tokyo 103-8515, JAPAN

*** Railway Technical Research Institute, ajiki@ rtri.or.jp

Power Supply Technology Division

2-89-38, Hikari-cho, Kokubunji-shi, Tokyo 185-8540, JAPAN
}

simulation using PSCAD/EMTDC but also a downscaled experimental devise.

Besides our proposal, the application of the power devices to the switchgears of the changeover section (static changeover section system) has been proposed and under development ${ }^{(7)}$. One of the advantages of our proposal is that our system is able to avoid the instantaneous power failure completely.

\section{Power System of Shinkansen Train}

2.1 Outline of the System As the Shinkansen train is driven by a single phase alternating electric power, three phase electric power from an electric power company should be converted to a single phase voltage at substations. To reduce the influence of the three phase unbalances to the upstream power system, a special transformer such as Scott connected transformer is applied and three phase voltage is converted to two single phase voltages, those are Mphase (main phase) and T-phase (teaser), whose phase difference is 90 degree each other. Because of the phase difference, it is impossible to connect M-phase and T-phase directly. In Shinkansen power feeding system, the phase-tophase changeover sections are built in front of substations (SS) and sectioning posts (SP) which are located between the substations. The circuit map of the A.C. power system for Shinkansen is shown in Fig. 1. Generally, the interval of the adjacent substations is about 50 or $60 \mathrm{~km}$. The AT (auto transformer) power feeding system is applied to the Shinkansen power system and the electric circuit is consist of the trolley, the feeder and the rail while only a trolley phase is described in this figure. 


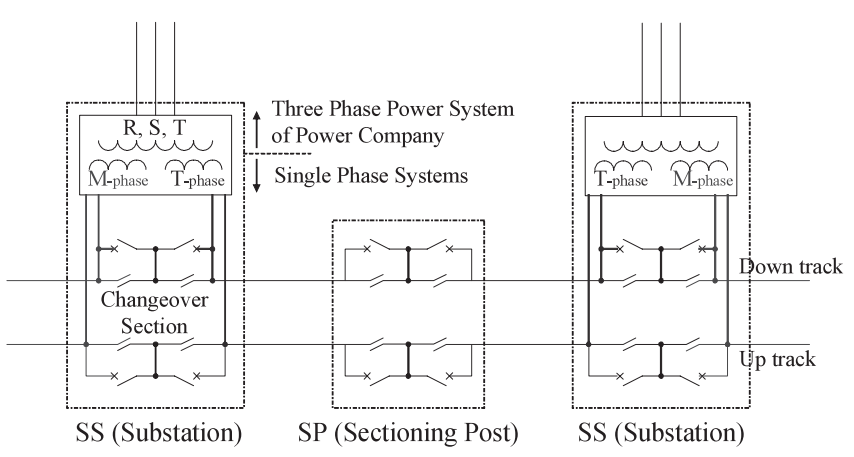

Fig. 1. Feeding network map of Shinkansen AC power system

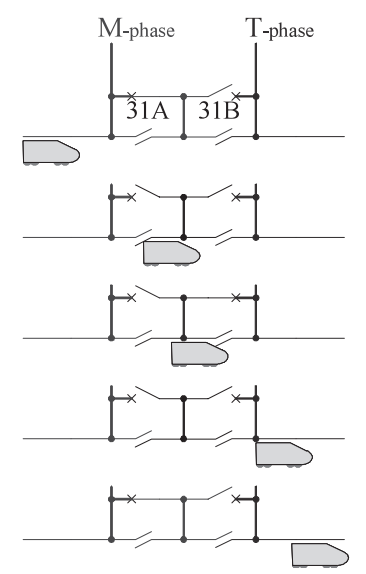
Procecure 1: Switchgear (31A) has been
closed and changeover section is connected
to M-phase.

Procecure 2: By detecting the progress of the train into the section, (31A) is opened.

Procecure 3: $300 \mathrm{~ms}$ later, (31B) is closed and the section is connected to T-phase.

Procecure 4: About 30s later, the train passes through the section completely and (31B) is opend.

Procecure 5: 300ms later, (31A) is closed

again and section is connected to M-phase to prepare for the following train.

Fig. 2. Procedure of the switchgear function at changeover section

2.2 Changeover Section As shown in Fig. 1, the phase different two single phases (M-phase and T-phase) are faced at the changeover section in front of the substation. In front of the sectioning post, on the other hand, the power line from different substation is faced.

The trolley (contact wire) of the changeover section is connected to one of the two faced power sources by switchgear, and the connected power source is changed instantaneously from one to the other. Such a sequence is executed while the train stays in the changeover section and after the train passes away from the changeover section. The instantaneous power failure during the procedure continues about $300 \mathrm{~ms}$ generally, and the length of the changeover section is about $1 \mathrm{~km}$. The example of the procedure of the switchgear function at changeover section is shown in Fig. 2.

\section{Closing Surge at Changeover Section}

3.1 Frequent Work of Arrester At Shin-Tabata substation (SS) on Tohoku Shinkansen Line, it is reported that the lightning arrester for the changeover section works frequently. Judging from the number of the discharge counter of the arrester, it works more than 10 times at most and about 5 times on the average per a day. As the ground-fault accident of the arrester happened at Shin-Tabata SS in 2001, it becomes a slight cause of the anxiety for daily maintenance and clarifying of the phenomenon is strongly required. The detail of phenomena is described in Ref. (1).

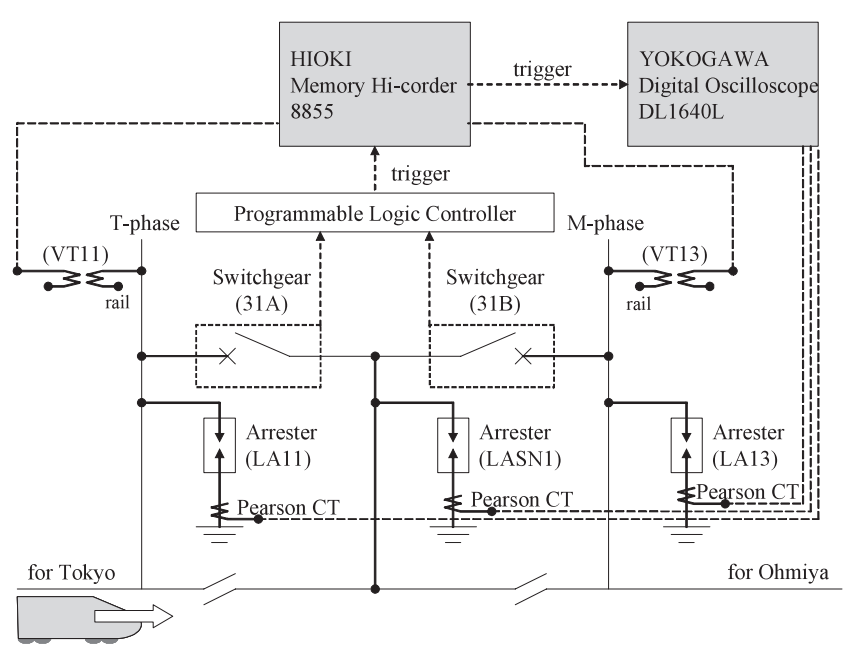

Fig. 3. Configuration of the measuring system

3.2 Measurement of the Discharge Current To elucidate the phenomena, the discharge currents of the arresters were measured at Shin-Tabata SS during the business operational hours. The configuration of the measuring system is shown in Fig. 3. Fig. 4 shows the photographs of the lightning arresters at Shin-Tabata SS and the mounted CT for discharge current measurement. The special current transformers for high speed discharge current (Pearson model325 ) were set on the groundings of arresters for T-phase for Tokyo (LA11), changeover section (LASN1) and M-phase for Ohmiya (LA13), respectively. To confirm the relation between the discharge of arresters and the work of switchgears, the state information of the switchgears (31A and 31B) is acquired from the protection system (PLC: programmable logic controller). The outputs of the voltage transformers of trolley of T-phase (VT11) and M-phase (VT13) were also recorded. The phenomenon was so fast, however, that it was impossible to know the instantaneous voltage level by these built-in VTs. These outputs were utilized to know the opening and closing timing of the switchgears and to know the voltage difference between the contacts of switchgear just before the closing of the switchgear. The measuring system was triggered by the switchgear information from PLC system.

During the field measurement for three days, the works of the arrester were captured 22 times and it was confirmed that most of them (20 times out of 22) happed when the switchgear 31A was closed without train load ("Procedure 5 " in Fig. 2). The typical waveforms of the discharge current are shown in Fig. 5.

As a transient surge phenomenon concerning to the changeover section, a surge caused by the high frequency reignition oscillation in vacuum switchgear was investigated in detail more than 10 years ago ${ }^{(2)}$ by a group of RTRI (Railway Technical Research Institute). The phenomenon happened, however, when the switchgear was closed with train load ("Procedure 3" in Fig. 2). So that, the surge phenomenon which leads to the arrester discharge at Shin-Tabata SS was different from the previously reported one.

Judging from Fig. 5, the discharge current was observed only in the grounding of the arrester for changeover section and it continues for about 10 micro seconds with the value of 


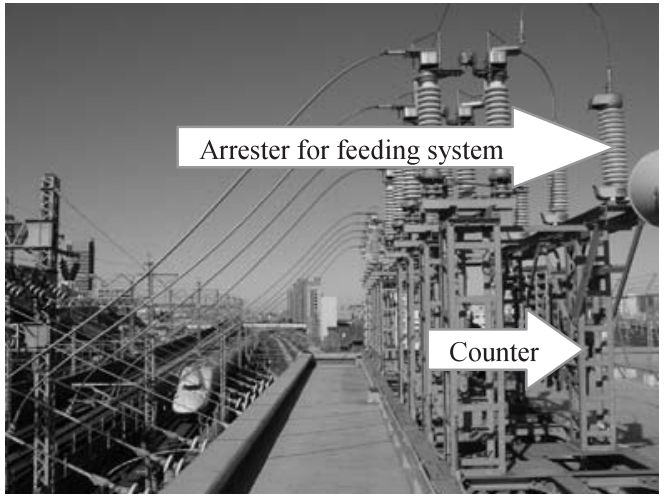

(a) Arresters of the feeding system at Shin-Tabata SS

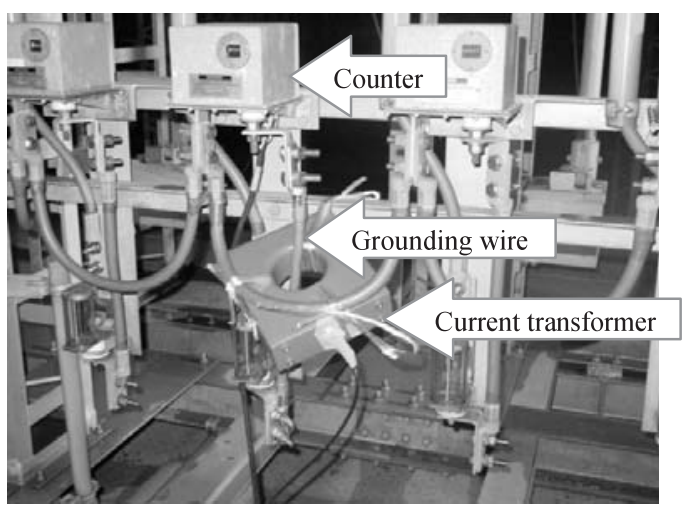

(b) Mounted CT and arresters counters

Fig. 4. Photos of the measurements

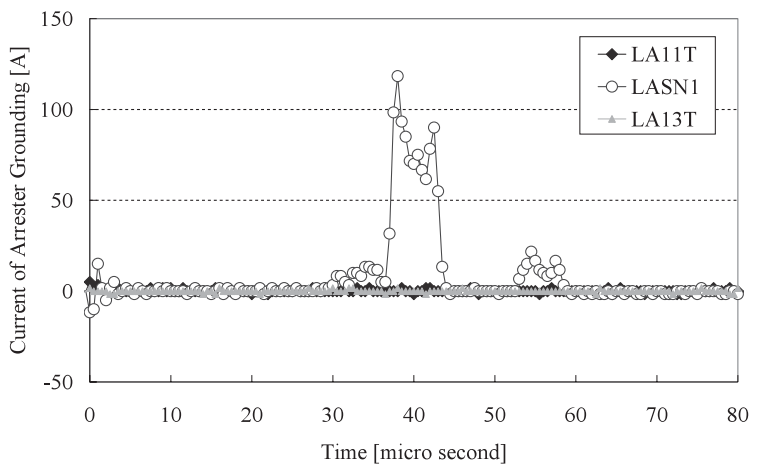

Fig. 5. Examples of the discharge current waveform

$100 \mathrm{~A}$. The dispersed energy during such a slight discharge is up to $100 \mathrm{~J}$ and it is estimated that it causes no damage or stress for arrester as the temperature rise caused by the discharge is roughly calculated to be 0.6 degree centigrade. The frequency of the work is, on the other hand, extremely high compared to the arresters for general power system of power companies, and the influence of such a frequent arrester work should be carefully tested in the future.

\subsection{Measurement of the Transient Surge Voltage}

To scrutinize the tendency of the closing surge without a train load, the feeding circuit of Shinkansen was energized during non-business hours and the switchgears were operated sequentially to realize the a transient surge phenomena.

Fig. 6 shows a typical voltage waveform when a large transient voltage was observed. The zoomed waveform of the surge wave-front in Fig. 6 is also shown in Fig. 7. The

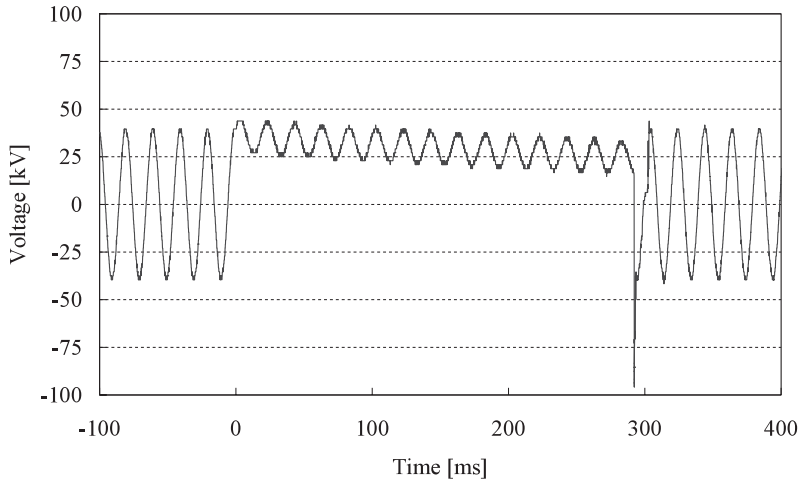

Fig. 6. Typical voltage waveform with a large overvoltage during the sequential switching

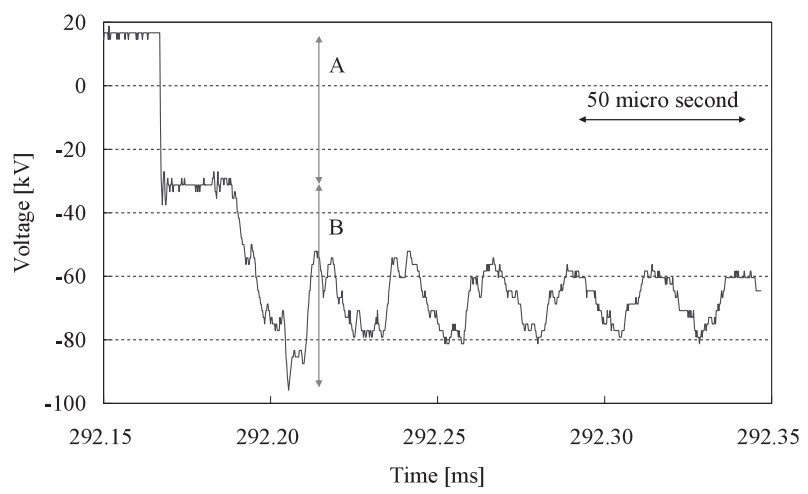

Fig. 7. Zoomed waveform of the wave-front in Fig. 6

Table 1. Over-voltage tendency

\begin{tabular}{c||c|c|c|c|c||c}
\hline Voltage[kV] & -20 & $20-40$ & $40-60$ & $60-80$ & $80-$ & Total \\
\hline \hline Frequency[times] & 11 & 10 & 15 & 7 & 7 & 50 \\
\hline Percentage[\%] & 22 & 20 & 30 & 14 & 14 & 100 \\
\hline
\end{tabular}

statistical over-voltage values are classified in Table 1.

In Fig. 6, one switchgear of changeover section was opened at $t=0 \mathrm{~ms}$ and another one was closed at around $t=$ $292.17 \mathrm{~ms}$. During the time between $t=0 \mathrm{~ms}$ and $t=$ $292.17 \mathrm{~ms}$, both switchgears are opened and the trolley of the changeover section was electrically floated. Because of the electrostatic induction, the DC biased AC voltage was monitored during this period. Judging from Fig. 7, the rise of the over-voltage began about 20 micro second after the first voltage step change at $t=292.17 \mathrm{~ms}$. At Shin-Tabata SS, the changeover section is built about $3 \mathrm{~km}$ away from the substation and it takes about 20 micro second for the traveling voltage wave to get there and back. The edges of the changeover section are electrically opened and the surge voltage is doubled and reflected there based on the traveling wave theory. So, it is possible to surmise that such an enhanced reflection voltage wave causes the frequent discharge of the arrester at changeover section. As shown in Table 1, the over-voltage may come to $100 \mathrm{kV}$ which will cause $100 \mathrm{~A}$ discharge current according to the non-linear voltage to current characteristic of the arrester and it is consistent with the results of the discharge current measurement.

Fig. 8 shows the relations between the voltage difference 


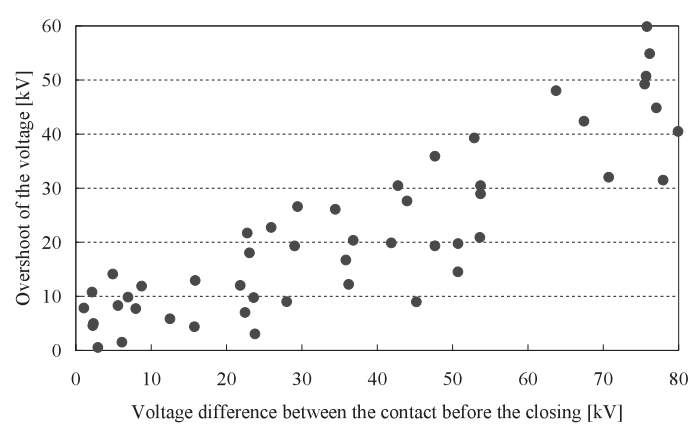

Fig. 8. Relation between the voltage difference of the switchgear contacts and the transient overshoot

of the contacts just before the closing of switchgear (A in Fig. 7) and the overshoot of the voltage (B in Fig. 7) based on the 50 times measurements without train loads at ShinTabata SS shown in Table 1. It should be paid attention that the amplitudes of the overshoot voltage are plotted in Fig.8, while the peak value of the over-voltage is counted in Table 1 . As shown in Fig. 8, there is a strong correlation between the voltage difference before the closing and the overshoot voltage after the closing. The reflection wave should be doubled theoretically and it may be dull during the transmission and the about 1.5 times wave was observed at SS according to our measurements.

3.4 Measures to the Surge Phenomena The necessity of the measures to this closing surge phenomena have to be judged taking the results of the arrester deterioration investigation into account from the practical points of view. If such slight but frequent discharges of the surge arrester leads to the arrester deterioration, the counter measure should be required in the future.

One solution to avoid such a transient phenomena is controlling the closing timing of the switchgear. By this method, the influence of the inrush current may be also redued. The closing control technology of the switchgear has recently reported $^{(5)}$ and the investigation of the static changeover section by RTRI and JR Central group ${ }^{(6)}$ will be also instructive.

Apart from such a technology on the extension of the conventional changeover section structure, a novel power system, named CSPS, is proposed in the following chapter. It is necessary to say, in advance, that the total cost of the proposed system will be so expensive that the realization of the system is difficult in the near future. The basic characteristics of the system, however, are confirmed in this paper. It has to be also clearly mentioned that the reducing the closing surge at changeover section was not the main purpose to introduce CSPS. It is one of the expected advantages of CSPS and, to the contrary, applying CSPS to the changeover section is one of the solutions to avoid the closing surge.

\section{Changeover Section Power System}

4.1 Problems of the Changeover Section In the conventional changeover section, a sudden change of the power source causes the transient voltage surge as mentioned in Chapter 3 . In addition to this, there are some disadvantages in the conventional changeover section system as follows: necessity of the careful maintenance of the switchgears because of the frequent mechanical work, possibility of short circuit
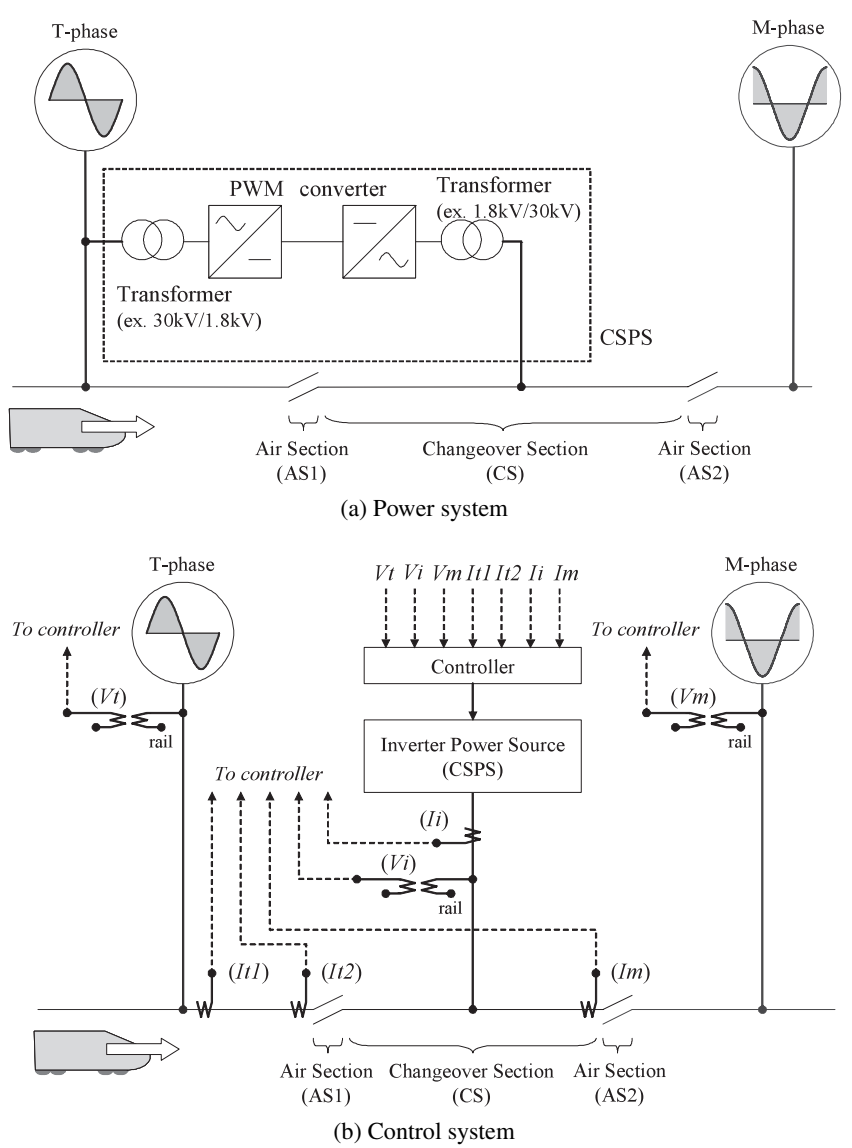

Fig. 9. Configuration of CSPS

faults between the contacts, probability of high inrush current depending on the closing timing, and so on. Furthermore, in the future, the latitude of the changeover time will not be enough when the train runs though the changeover section shorter than $1 \mathrm{~km}$ if the high speed running up to $400 \mathrm{~km} /$ hour is supposed.

4.2 Changeover Section Power System To avoid such disadvantages, a novel inverter controlled power system for changeover section, that is the CSPS, was proposed ${ }^{(4)}$. The configuration of the system is shown in Fig. 9. This figure and the following explanation are supposing the movement of the train from T-phase power source to M-phase power source.

In this system, the train is able to pass the changeover section without the switching of the mechanical switchgears. The voltage of the changeover section is controlled by VVVF inverter and the phase is shifted from T-phase to M-phase gradually while the train is passing through the changeover section. To realize the fast and smooth passing of the train through the section, the following three proposed functions are necessary:

$\checkmark$ the phase and voltage shift control

$\checkmark$ the load transfer control

$\checkmark$ the output restriction control

In the voltage and phase shift control, the phase of the section is synchronized to the T-phase before the train comes into the section. When the train comes into the section completely, it is gradually shifted and synchronized to the Mphase in a few seconds. While the changeover section is 
synchronized to the T-phase, the amplitude and the phase of the changeover section voltage are a little bit shifted to those of the T-phase. When the train comes to the changeover section and the pantograph makes the air section short, the slight differences between the T-phase and the changeover section diminishes and the approach of the train into the changeover section is electrically detected. In case of the multi pantographs train, the following pantograph is detected by the signal from the CT to measure It 1 and the beginning of the phase shift to synchronize to M-phase is suspended.

In the load transfer control, the train load is transferred from the backward power source to the forward power source by controlling the amplitude and phase of the inverter output when the pantograph comes to the air section and connects these power sources. By virtue of this control, it is possible to avoid the generation of the strong arc when the pantograph leaves the trolley line of the backward power source. As shown in the following investigation, it is difficult to transfer the load completely and it should be decreased as low as possible. Practically, it is necessary to detect the load current roughly to realize this control and such a technology is premature now. As it is not necessary to know the exact current value, this problem will be solved in the future. The application of the optical CT, for example, to the messenger wire will be one of the possible solutions.

The output restriction control is the control which makes the output of the CSPS stable when two pantographs with bus connection stride across the air section. Because of the bus, it is impossible to shift the phase of the inverter output though the CSPS tried to perform the voltage and phase shift control. In this control, the controller judges the situation that two pantographs stride across the air section when the current of the air section is zero while the slight difference diminishes and the output of the CSPS is restricted under this condition.

The control sequence when the train which has two pantographs with bus comes into the changeover section from T-phase is summarized in Fig. 10. As shown in Fig. 10, the control modes are selected based on the information of $V t$ and $V i, I t 1$ and $I t 2$. When the train is before the air section, the changeover section is almost synchronized to the T-phase by virtue of the voltage and phase shift control. The arrival of the first pantograph to the air section is detected by the diminish of the voltage difference between $V t$ and $V i$ and the load transfer control is applied. If there is no second pantograph, the complete entrance of the pantograph into the changeover

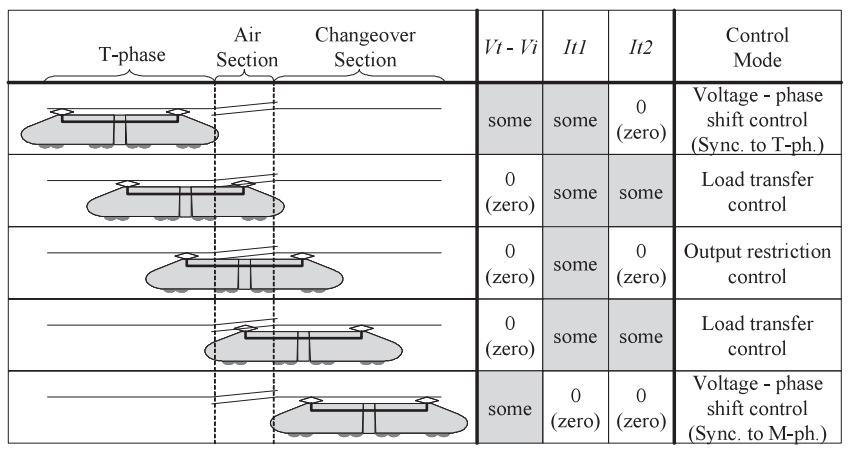

Fig. 10. Example of the control sequence sectionis detected as $I t 1$ and It 2 become 0 when the pantograph leaves the T-phase trolley. In the case shown in Fig. 10, however, there is a second pantograph which is electrically connected to the first pantograph. Such condition is detected by the validation of the following condition: $V t=V i$, It 1 has a value and $I t 2=0$. Then the output restriction control is applied to prepare for the following second pantograph and make the output of CSPS stable. After detecting the current, It 2 , the load transfer control is applied again and finally the voltage and phase shift control is performed to synchronize to the M-phase when the all the pantographs comes into the changeover section completely. When the train goes into Mphase from changeover section, the CSPS is controlled using the information of $V i$ and $V m, I i$ and $I m$, in the same way.

In case that there are some pantographs and some of them are independent from the others, they are treated as different train and the CSPS respond quickly.

One of the other possible ways to realize a system without power failure is the introduction of the 3phase/single phase transformation using power electronics devices. In this way, the changeover section in front of the substation is able to be omitted. Concerning to the system capacity, however, the converter have to feed the electric power to all the trains existing in the whole feeding area in this method and have to be designed supposing the continuing power feeding. The capacity of the converter will be same as the conventional Scott transformer, that is about $100 \mathrm{MVA}$ or the more. Contrary to this, the proposed CSPS system feeds the power only to the train in the changeover section. The capacity of the converter will be about $20 \mathrm{MVA}$ and the short time rated capacity should be applied which will result in the total cost reduction.

\subsection{Computer Simulation Results The electrical} circuit simulations were performed using PSCAD/EMTDC to confirm the basic algorithm of the CSPS. Fig. 11 shows the simulation circuit. The configuration of the current collection system of the train is supposed to be two pantographs with bus as explained in Fig. 10. The movement of the train is simulated by handling the switches sequentially to make the moderate electrical circuit. The latter number of each switch

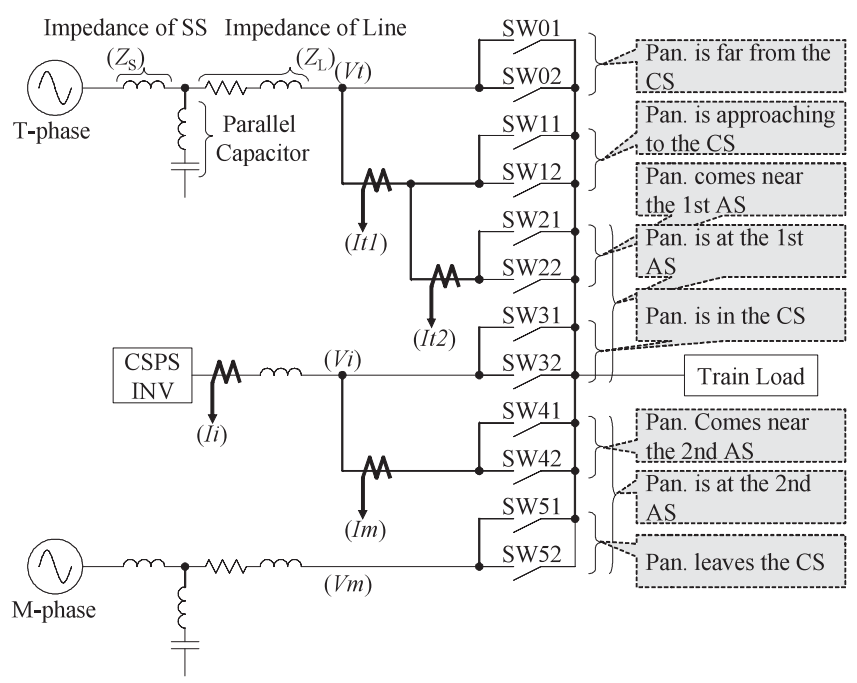

Fig. 11. Simulation circuit (AS: Air Section, CS: Changeover Section) 

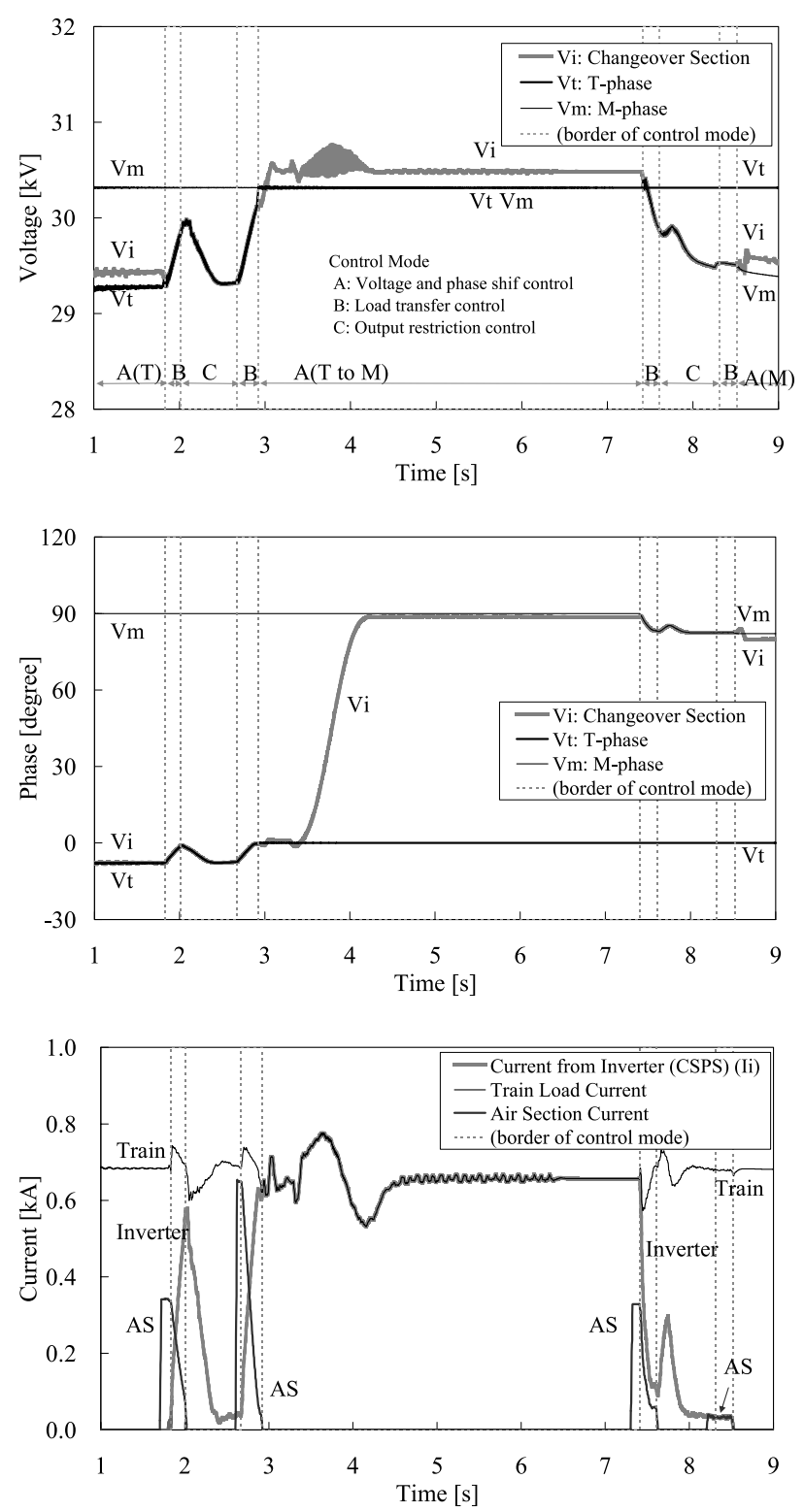

Fig. 12. Simulation results

indicates the pantograph number and the former one indicates the position of that pantograph, respectively. The relation between the closed switch number and the correspondent location of the pantograph is also shown in the same figure.

The impedance of the substation including the upper system is set to be $\mathrm{j} 1.7 \%$ or $\mathrm{j} 3.5 \%$ referring to the practical system at $10 \mathrm{MVA}$. The impedance of the line is $0.04+$ $\mathrm{j} 0.15 \mathrm{ohm} / \mathrm{km}$ and the length of line $(L)$ is chosen as 0,30 and $60 \mathrm{~km}$ which correspond to the changeover section at $\mathrm{SS}$, SP and when extended feeding is applied, respectively. The train is modeled as a constant power load up to $20 \mathrm{MW}$ from powering to regenerating. When the parallel capacitor at SS is modeled, it is set to be $6 \mathrm{MVA}$ with $12 \%$ reactor.

The simulations results are shown in Fig. 12 under the following condition: $Z_{S}$ and $L$ are set to be $\mathrm{j} 1.7 \%$ and $30 \mathrm{~km}$ (supposing sectioning post), the train is powering at $20 \mathrm{MW}$, the substation has no parallel capacitor, respectively. The time evolutions of the root mean square values are shown in these figures. The length of the changeover section, air

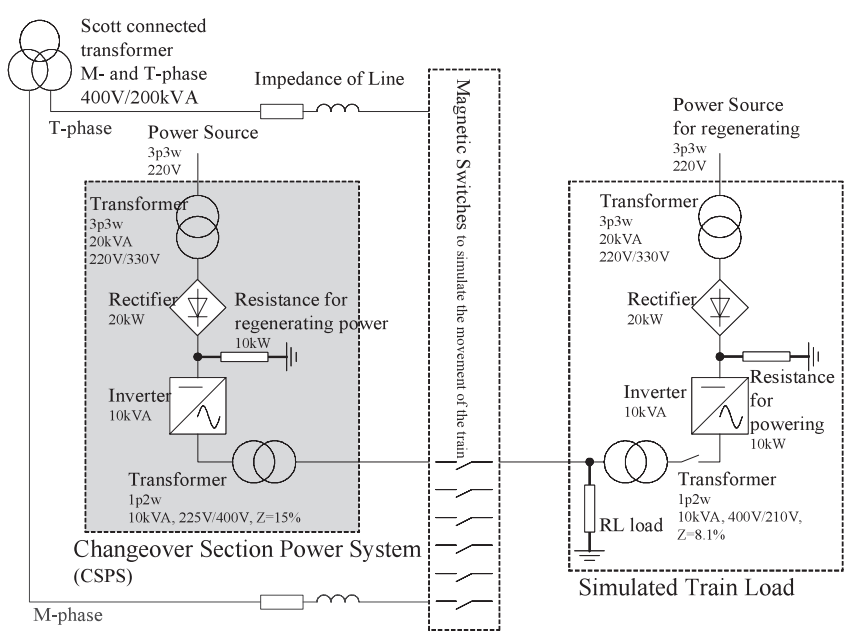

Fig. 13. Circuit figure of the mini-model

Table 2. Specifications of the mini-model

\begin{tabular}{|c|c|c|c|}
\hline \multicolumn{2}{|l|}{ PWM inverter } & \multicolumn{2}{|l|}{ Train load } \\
\hline Phase number & Single phase & Power factor & 0.98 or 1.00 \\
\hline Capacity & $400 \mathrm{~V}, 10 \mathrm{kVA}$ & $\begin{array}{l}\text { PWM } \\
\text { converter }\end{array}$ & $400 \mathrm{~V}, 10 \mathrm{kVA}$ \\
\hline Switching Freq. & $3.6 \mathrm{kHz}$ & Resistance & $15 \mathrm{ohm}, 10 \mathrm{~kW}$ \\
\hline Resistance & $18 \mathrm{ohm}, 10 \mathrm{~kW}$ & \multicolumn{2}{|c|}{ Power source of feeding system } \\
\hline Power Device & IGBT & \multirow{2}{*}{$\begin{array}{l}\text { Scott connected } \\
\text { transformer }\end{array}$} & \multirow{2}{*}{$\begin{array}{l}400 / 400 / 400 \mathrm{~V}, \\
400 / 200 \mathrm{kVA}\end{array}$} \\
\hline Others & & & \\
\hline Switch & Magnetic Sw. & Frequency & $50 \mathrm{~Hz}$ \\
\hline
\end{tabular}

section and the interval of two pantographs is supposed to be about $500 \mathrm{~m}$ (much shorter than the actual changeover section), $30 \mathrm{~m}$ and $50 \mathrm{~m}$, respectively and the speed of the train is assumed to be $400 \mathrm{~m} /$ hour to prove the ability of the system.

As shown in Fig. 12, the output of the CSPS is moderately controlled by the voltage and phase shift control, the load transfer control and the output restriction control. Concerning to the residual current when the pantograph leaves the air section, it is decreased to be $15.1 \mathrm{~A}$ at $t=2.92 \mathrm{~s}$ and $29.6 \mathrm{~A}$ at $t=8.51 \mathrm{~s}$ by virtue of the load transfer control. The phase of the inverter output is gradually shifted from T-phase to M-phase from $t=3.3 \mathrm{~s}$ to $4.3 \mathrm{~s}$ by detecting the complete entrance of the train into the changeover section.

4.4 Experimental Results using Mini-Model The validity of each control method was also experimentally confirmed using the downsized mini-model. Here, the train that had independent two pantographs, namely without bus, was supposed, so that the effect of the output restriction control was not evaluated. The circuit figures of the CSPS and the simulated train load are shown in Fig. 13 and the specification of the mini-model is shown in Table 2. The movement of the train was simulated by handling of the magnetic switches by a sequencer like above mentioned computer simulation. The converter of the train load was used to confirm the influence of the re-powering.

The current waveforms of the mini-model experiment supposing the $400 \mathrm{~km} / \mathrm{h}$ running with $10 \mathrm{~kW}$ powering load is shown in Fig. 14. Only the waveforms when the train comes into the changeover section are shown in this figure. The functions of the proposed control methods are confirmed 


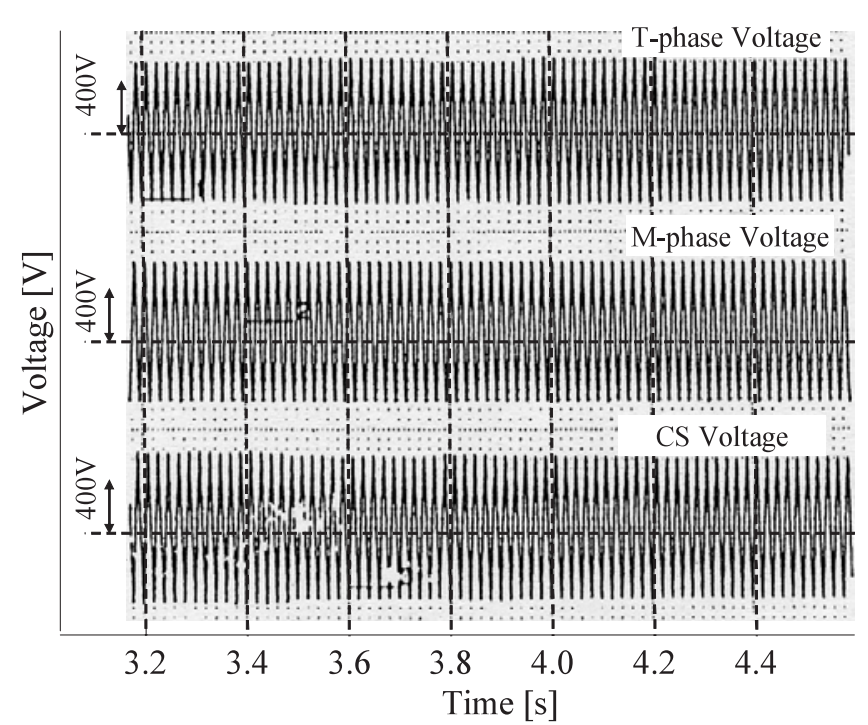

(a) Voltage waveforms

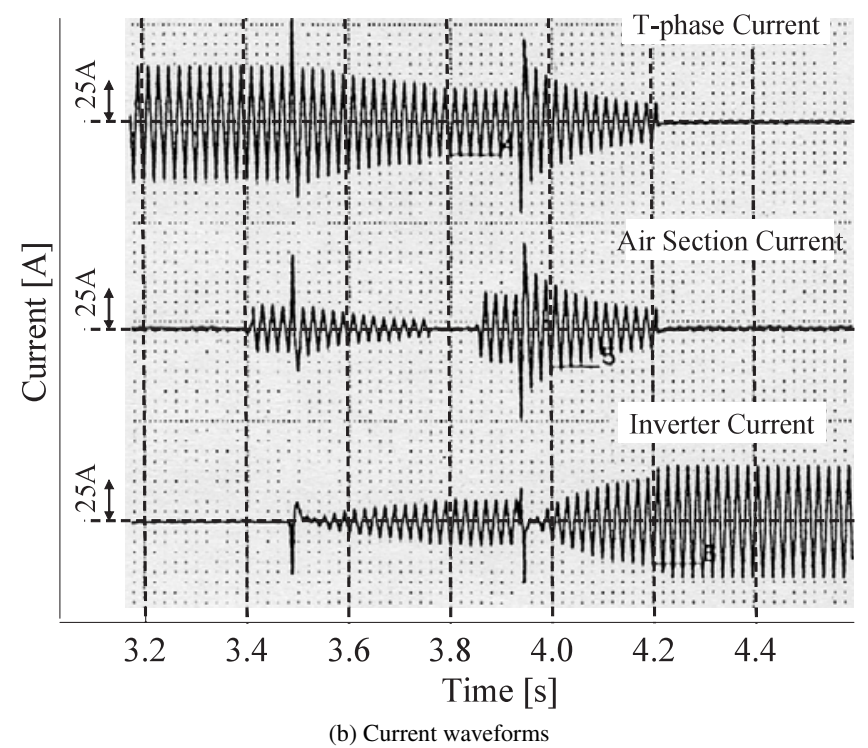

Fig. 14. Results of mini-model experiments

through the mini-model experiments as follows:

- The load current from the rear power source to the train through pantograph in the air section was decreased before leaving of the pantograph from the air section ( $t=3.76 \mathrm{~s}$ and $t=4.20 \mathrm{~s}$ ) by virtue of the load transfer control. Because of this control, the damage of the trolley by the arc will be lessened.

- The approaching of the second pantograph was automatically detected and the CSPS prepared for that. When the first pantograph enters into the changeover section completely, the controller does not begin the phase shift control to synchronize to the M-phase.

- The phase of the CSPS output was gradually shifted from T-phase to M-phase stably after all the pantographs had entered the changeover section completely. The entrance of the all pantographs in the changeover section was automatically and moderately detected $(t=4.25 \mathrm{~s})$.

A problem brought by the experiments is that the transient over-current were observed when the pantograph had just connected to the CSPS power source at $t=3.50 \mathrm{~s}$ which was not seen in computer simulations. Furthermore, the limit of the residual current which may cause the arc and make the trolley damaged is not clear for the practical use. They should be discussed and solved in the future.

\section{Conclusions}

In this paper, the transient surge phenomena caused by the closing of the vacuum switchgear at Shinkansen changeover section were carefully reported. According to the surge voltage measurements, the surge voltage is brought by the reflection at the open terminal and strongly depends on the voltage difference between the contacts of the switchgear just before the closing. The amplitude of the surge voltage reaches to be $100 \mathrm{kV}$ and such a transient voltage is suppressed by the lightning arresters moderately in the practical field. The frequency of the working of arrester is, however, so often that the influence of the frequent work of the surge arrester will be investigated in the future. If it leads to the stress or damage of the arrester, some measures should be applied.

As one of the solutions to avoid such a transient phenomena in Shinkansen changeover section, a novel power supply system for changeover section was proposed. The basic functions of the system were confirmed by the computer simulations and the mini-model experiments. The validity of the following control methods for the proposed power supply system was proved through the investigations:

$\checkmark$ the voltage and phase shift control

$\checkmark$ the load transfer control

$\checkmark$ the output restriction control

Needless to say, the cost reduction will be the most important and the toughest problem for the practical introduction of the proposed system.

\section{Acknowledgment}

The onsite measurements of the surge phenomena were realized by strong supports from maintenance staffs of JR East. Especially we wish to acknowledge devoted contributions of Mr. H. Akazawa, Mr. M. Nagao, Mr. Y. Kojima, Mr. T. Iwata, Mr. M. Hashimoto, Mr. T. Ishii, Mr. M. Ogiwara, S. Kikuchi and Mr. M. Ohmori, respectively. The discussion about surge phenomena with Mr. K. Arai, Prof. S. Kojima, Mr. H. Inoue and Mr. I. Ideno were valuable to solve the problems. The surge voltage measurements are supported by the staffs of RTRI, those are Mr. S. Sugai and Mr. T. Aihara. The development of the CSPS is based on the basic proposal of the system by the engineers of Meidensha Corporation. Especially we wish to acknowledge valuable discussion with Mr. H. Watanabe, Mr. M. Ono, Mr. H. Machida and Mr. T. Shibuya. They carefully assisted and strongly contributed to our development intently.

(Manuscript received May 9, 2005,

revised Dec. 28, 2005)

This paper was presented at IPEC-Niigata 2005, and approved for publication in the IEEJ Transactions on Industry Applications Society.

\section{References}

( 1 ) H. Hayashiya et al.: "Discharge phenomena of lightning arrester of Shinkansen changeover section", The papers of Tech. Meeting on trans. and electric railway, IEE Japan, No.TER-04-38, pp.41-45 (2004) (in Japanese) 
( 2 ) Y. Mochinaga et al.: "Occurrence and countermeasures for high-frequency re-ignition oscillation in vacuum switch for Shinkansen changeover section", T. IEE Japan, Vol.111-D, No.9, pp.777-784 (1991-9) (in Japanese)

( 3 ) K. Ajiki et al.: "Analysis of the phenomenon associated with the short circuit of Shinkansen changeover switch and development of new type protective relay", T. IEE Japan, Vol.122-B, No.4, pp.498-505 (2002-4) (in Japanese)

(4) Y. Ueda et al.: "Basic study on a power supply system for Shinkansen changeover section", 2004 National Convention Record of IEE Japan, Vol.5, No.143, pp.208-209 (2004) (in Japanese)

( 5 ) H. Hohyama et al.: "Reduction of switching surge by controlled shunt reactor switching of gas circuit breaker", T. IEE Japan, Vol.124-B, No.2, pp.267-273 (2004-2) (in Japanese)

( 6 ) K. Ajiki et al.: "Investigation of Shinkansen static changeover switch capable of suppression of exciting inrush current into transformer on train", $T$. IEE Japan, Vol.121-D, No.3, pp.340-346 (2001-3) (in Japanese)

( 7 ) T. Saito et al.: "A control method to suppress exciting inrush-current of traction transformers by the static change-over switch for the Shinkansen train", 2002 National convention record, IEEJ, No.5-194, pp.265-266 (2002) (in Japanese)

Hitoshi Hayashiya (Member) was born on June 28th, 1970. He grad-

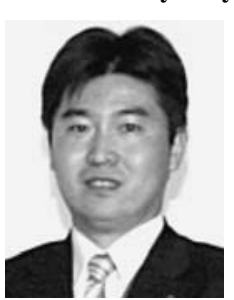
uated from the department of electrical engineering, the University of Tokyo in 1999, worked for the University of Tokyo engaging in the studies of magnetic levitation, linear drive systems and fusion plasmas. He has been worked for the R\&D center of JR East group since 2002. Member of IEEJ and The Japan Society of Applied Electromagnetics. Ph.D.

Yoshinobu Ueda

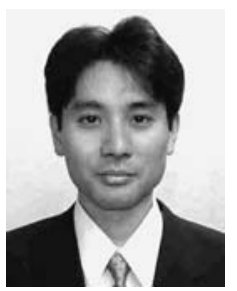

(Member) was born on December 10th, 1973. He graduated from the department of electrical engineering, the University of Tokyo in 2001. His specialty at University was also fusion plasmas. He has been worked for Meidensha Corporation since then and mainly engages in the development of power electronics application for power system and railway system. Ph.D.
Koji Ajiki (Member) was born on March 26th, 1956. He graduated

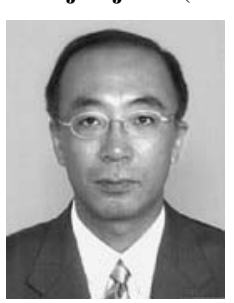
from the department of applied physics, the Science University of Tokyo in 1979 . He has worked for Japan National Railway and Railway Technical Research Institute since 1979. He mainly engages in research and development concerning to the high voltage and insulation technology of railway power system.

Hideo Watanabe (Member) was born on January 25th, 1953. He

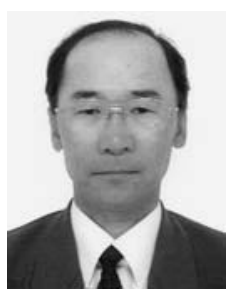
graduated from the department of electrical engineering, Hokkaido University. He entered Japan National Railway in 1976 and has been worked for Meidensha Corporation since 1989.

Masato Ando (Member) was born on July 22nd, 1971. He graduated

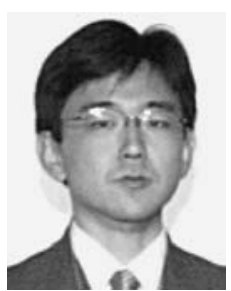
from the department of electrical engineering, Tokyo Institute of Technology in 1995 and has been worked for JR East since then. He was a member of Railway Technical Research Institute from 1999 to 2002 and engaged in the research of AC power system for railway transportation. He works at the head office of JR East.

Makoto Nakajima (Non-member) was born on August 26th, 1977.

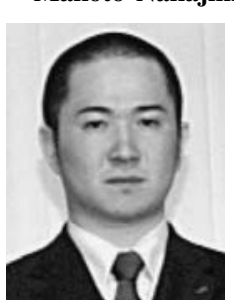

He has been worked for JR East since 1997 and now works at Tokyo Substation Technology Center, Tokyo Branch Office. He engages in a daily maintenance of substations for AC and DC railway system. 